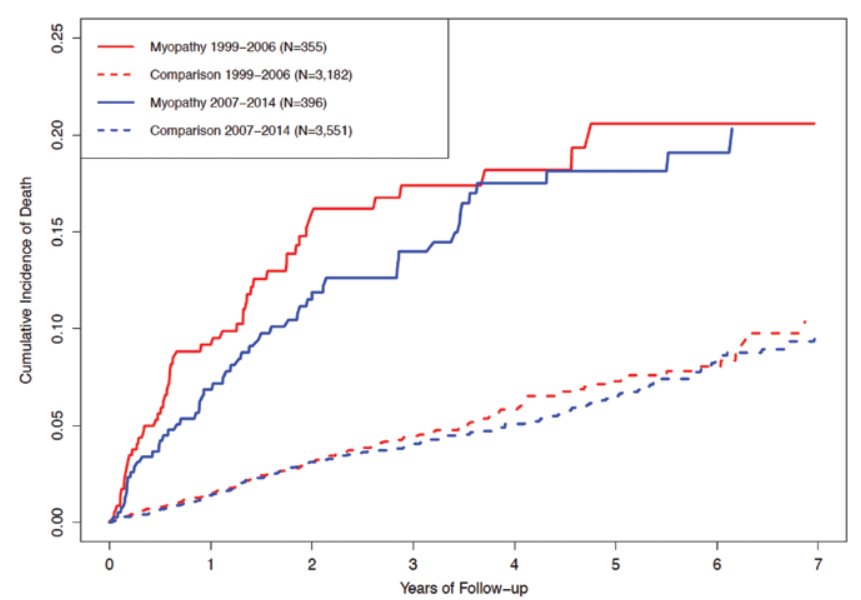

Figure. Cumulative mortality of patients with idiopathic inflammatory myopathy and matched controls without IIM in early versus late cohorts (1999-2006 versus 2007-2014).

Disclosure of Interests: Kristin D'Silva: None declared, Leo Lu: None declared, Alexis Ogdie Grant/research support from: Pfizer to Penn, Novartis to Penn, Amgen to Forward/NDB, Consultant of: Abbvie, Amgen, Bristol-Myers Squibb, Celgene, Corrona, Janssen, Eli Lilly, Novartis, Pfizer, Antonio Aviña: None declared, Hyon Choi Grant/research support from: Ironwood, Horizon, Consultant of: Takeda, Selecta, Horizon, Kowa, Vaxart, Ironwood DOI: 10.1136/annrheumdis-2020-eular.2230

\section{OP0248 $\quad$ PREMATURE MORTALITY BURDEN FOR SYSTEMIC SCLEROSIS: NATIONWIDE POPULATION-BASED STUDY}

E. Yen ${ }^{1}$, D. Singh ${ }^{1}, \mathrm{M} . \mathrm{Wu}^{1}$, R. Singh ${ }^{1} .{ }^{1}$ UCLA, Los Angeles, United States of America

Background: Premature mortality is an important way to quantify disease burden. Patients with systemic sclerosis (SSc) can die prematurely of disease, however, the premature mortality burden of SSc is unknown. The years of potential life lost (YPLL), in addition to age-standardized mortality rate (ASMR) in younger ages, can be used as measures of premature death.

Objectives: To evaluate the premature mortality burden of SSc by calculating: 1) the proportions of SSc deaths as compared to deaths from all other causes (non-SSc) by age groups over time, 2) ASMR for SSc relative to non-SSc-ASMR by age groups over time, and 3) the YPLL for SSc relative to other autoimmune diseases.

Methods: This is a population-based study using a national mortality database of all United States residents from 1968 through 2015, with SSc recorded as the underlying cause of death in 46,798 deaths. First, we calculated the proportions of deaths for SSc and non-SSc by age groups for each of 48 years and performed joinpoint regression trend analysis ${ }^{1}$ to estimate annual percent change (APC) and average APC (AAPC) in the proportion of deaths by age. Second, we calculated ASMR for SSc and non-SSc causes and ratio of SSc-ASMR to nonSSc-ASMR by age groups for each of 48 years, and performed joinpoint analysis to estimate APC and AAPC for these measures (SSc-ASMR, non-SSc-ASMR, and SSc-ASMR/non-SSc-ASMR ratio) by age. Third, to calculate YPLL, each decedent's age at death from a specific disease was subtracted from an arbitrary age limit of 75 years for years 2000 to 2015 . The years of life lost were then added together to yield the total YPLL for each of 13 preselected autoimmune diseases. Results: $23.4 \%$ of all SSc deaths as compared to $13.5 \%$ of non-SSc deaths occurred at $<45$ years age in 1968 ( $p<0.001$, Chi-square test). In this age group, the proportion of annual deaths decreased more for SSc than for non-SSc causes: from $23.4 \%$ in 1968 to $5.7 \%$ in 2015 at an AAPC of $-2.2 \%(95 \% \mathrm{Cl},-2.4 \%$ to $-2.0 \%)$ for SSc, and from $13.5 \%$ to $6.9 \%$ at an AAPC of $-1.5 \%(95 \% \mathrm{Cl},-1.9 \%$ to $-1.1 \%$ ) for non-SSc. Thus, in 2015, the proportion of SSc and non-SSc deaths at $<45$ year age was no longer significantly different. Consistently, SSc-ASMR decreased from $1.0(95 \% \mathrm{Cl}, 0.8$ to 1.2$)$ in 1968 to $0.4(95 \% \mathrm{Cl}, 0.3$ to 0.5$)$ per million persons in 2015 , a cumulative decrease of $60 \%$ at an AAPC of $-1.9 \%(95 \%$ $\mathrm{CI},-2.5 \%$ to $-1.2 \%$ ) in $<45$ years old. The ratio of SSc-ASMR to non-SSc-ASMR also decreased in this age group (cumulative $-20 \%$, AAPC $-0.3 \%$ ). In $<45$ years old, the YPLL for SSc was 65.2 thousand years as compared to 43.2 thousand years for rheumatoid arthritis, 18.1 thousand years for dermatomyositis, 146.8 thousand years for myocarditis, and 241 thousand years for type 1 diabetes. Conclusion: Mortality at younger ages ( $<45$ years) has decreased at a higher pace for SSc than from all other causes in the United States over a 48-year period. However, SSc accounted for more years of potential life lost than rheumatoid arthritis and dermatomyositis combined. These data warrant further studies on SSc disease burden, which can be used to develop and prioritize public health programs, assess performance of changes in treatment, identify high-risk populations, and set research priorities and funding.

References:

[1] Yen EY....Singh RR. Ann Int Med 2017;167:777-785.

Disclosure of Interests: None declared

DOI: 10.1136/annrheumdis-2020-eular.2270

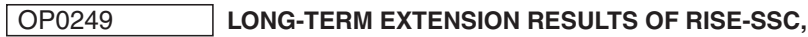 A RANDOMIZED TRIAL OF RIOCIGUAT IN PATIENTS WITH EARLY DIFFUSE CUTANEOUS SYSTEMIC SCLEROSIS (DCSSC)}

D. Khanna ${ }^{1}$, J. Pope $^{2}$, M. Matucci-Cerinic ${ }^{3}$, M. Kuwana ${ }^{4}$, C. Denton ${ }^{5}$, Y. Allanore ${ }^{6}$, M. Wosnitza ${ }^{7}$, M. E. Truchetet ${ }^{8}$, G. Szücs ${ }^{9}$, W. Stevens ${ }^{10}$, V. Steen ${ }^{11}$, C. Stagnaro ${ }^{12}$, V. Smith ${ }^{13}$, R. Silver ${ }^{14}$, E. Schiopu ${ }^{15}$, V. Riccieri ${ }^{16}$, F. Kramer ${ }^{7}$, S. Johnson ${ }^{17}$, O. Ishikawa ${ }^{18}$, T. Ishii ${ }^{19}$, E. Hachlla ${ }^{20}$, E. De Langhe ${ }^{21}$, L. Czirják ${ }^{22}$, R. Bečvárí ${ }^{23}$, T. Atsumi ${ }^{24}$, O. Distler ${ }^{25} .{ }^{1}$ Univ Michigan, Ann Arbor, United States of America; ${ }^{2}$ Univ Western Ontario, Ontario, Canada; ${ }^{3}$ Univ Florence, Florence, Italy; ${ }^{4}$ Nippon Med School, Tokyo, Japan; ${ }^{5}$ UCL, London, United Kingdom; ${ }^{6}$ Paris Descartes Univ, Paris, France; ${ }^{7}$ Bayer AG, Wuppertal, Germany; ${ }^{8}$ Bordeaux Univ Hosp, Bordeaux, France; ${ }^{9}$ Univ Debrecen, Debrecen, Hungary; ${ }^{10}$ St Vincent's Hosp, Melbourne, Australia; ${ }^{11}$ Georgetown Univ, Washington, United States of America; ${ }^{12}$ Univ Pisa, Pisa, Italy; ${ }^{13}$ Ghent Univ Hosp, Ghent, Belgium; ${ }^{14}$ Medical Univ South Carolina, Charleston, United States of America; ${ }^{15}$ Michigan Medicine Univ Hosp, Ann Arbor, United States of America; ${ }^{16}$ Sapienza Univ Rome, Rome, Italy; ${ }^{17}$ Univ Toronto, Ontario, Canada; ${ }^{18}$ Gunma Univ, Maebashi, Japan; ${ }^{19}$ Tohoko Univ, Sendai, Japan; ${ }^{20}$ Hôpital Claude Huriez, CHU, Lille Univ, Lille, France; ${ }^{21}$ Univ Hosp Leuven, Leuven, Belgium; ${ }^{22}$ Univ Pécs Medical School, Pécs, Hungary; ${ }^{23}$ Charles Univ, Prague, Czech Republic; ${ }^{24}$ Hokkaido Univ, Sapporo, Japan; ${ }^{25}$ Univ Hosp Zurich, Zurich, Switzerland

Background: RISE-SSc (NCT02283762) was a multicenter Phase Ilb trial of riociguat in pts with early (duration $\leq 18$ months) dcSSc and modified Rodnan skin score (mRSS) 10-22 units. Pts were randomized double-blind to placebo or riociguat $0.5-2.5 \mathrm{mg}$ t.i.d. for 52 weeks. The primary endpoint, mRSS change from baseline to Week (Wk) 52, did not reach statistical significance $(p=0.08$, riociguat vs placebo), but there were favorable trends in some other outcomes. Objectives: To present open-label long-term extension (LTE) results of RISE-SSc.

Methods: Pts who completed Wk 52 of double-blind therapy could enter LTE on riociguat. Endpoints included mRSS, adverse events (AEs), and serious AEs (SAEs)

Results: Of 60 pts randomized to riociguat and 61 to placebo, 42 (riociguat-riociguat group) and 45 (former placebo group), respectively, entered LTE. At LTE start, mean \pm SD mRSS was $16.4 \pm 3.2$ and $16.3 \pm 4.2$ units, and mean disease duration was $8.9 \pm 7.8$ and $8.9 \pm 5.8$ months, in the riociguat-riociguat and former placebo groups, respectively. Other demographics/disease characteristics were also comparable. Median duration of riociguat treatment was $1092 \mathrm{~d}$ in riociguat-riociguat pts and $649 \mathrm{~d}$ in former placebo pts. Throughout the study, mRSS decreased in both groups (Figure 1). From Wk 52 to last visit, mRSS fell by $-3.02 \pm 5.51$ in riociguat-riociguat patients and $-3.96 \pm 5.43$ in former placebo pts. Rates of $m R S S$ regression (decrease by $>5$ units and $\geq 25 \%$ from Wk 52 to last visit) and of \% declines in mRSS were similar in the two groups (Figure 2). mRSS progression (increase by $>5$ units and $\geq 25 \%$ from Wk 52 to last visit) occurred in 1 pt $(2 \%)$ in each group. During the entire study, rescue therapy agents were used in $15(36 \%)$ riociguat-riociguat pts and $17(38 \%)$ former placebo pts. AEs were reported from Wk 52 to last visit in 82 pts (94\%): 40 (95\%) riociguat-riociguat and 42 (93\%) former placebo. Most common AEs overall: nasopharyngitis (24\%), gastroesophageal reflux disease (17\%), diarrhea (15\%), and hypotension (14\%). AEs of special interest (dizziness, postural dizziness, or hypotension) occurred in 5 riociguat-riociguat pts (12\%) and 4 former placebo pts $(9 \%)$. SAEs were reported in $21(24 \%)$ pts: 10 (24\%) riociguat-riociguat pts and $11(24 \%)$ former placebo pts, with no SAE reported in $>1$ patient, no SAEs of special interest, and no deaths.

Conclusion: During LTE riociguat treatment, mRSS decreased in both groups from Wk 52 onwards and mRSS progression was uncommon. Riociguat had acceptable safety, similar to the main study, with no new safety signal. 